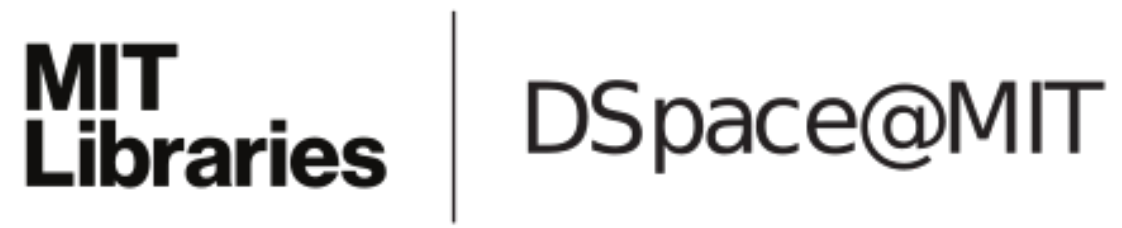

\author{
MIT Open Access Articles
}

Journey-based characterization of multimodal public transportation networks

The MIT Faculty has made this article openly available. Please share how this access benefits you. Your story matters.

Citation: Viggiano, Cecilia et al. "Journey-Based Characterization of Multi-Modal Public Transportation Networks." Public Transport 9.1-2 (2017): 437-461.

As Published: http://dx.doi.org/10.1007/s12469-016-0145-8

Publisher: Springer Berlin Heidelberg

Persistent URL: http://hdl.handle.net/1721.1/110697

Version: Author's final manuscript: final author's manuscript post peer review, without publisher's formatting or copy editing

Terms of Use: Article is made available in accordance with the publisher's policy and may be subject to US copyright law. Please refer to the publisher's site for terms of use. 


\title{
Journey-Based Characterization of Multi-Modal Public Transportation Networks
}

\author{
Cecilia Viggiano · Haris N. Koutsopoulos · \\ Nigel H.M. Wilson · John Attanucci
}

the date of receipt and acceptance should be inserted later

\begin{abstract}
Planners must understand how public transportation systems are used in order to make strategic decisions. Smart card transaction data provides vast, detailed records of network usage. Combined with other automatically collected data sources, established inference methodologies can convert smart card transactions into complete linked journeys made by individuals within the public transport network. However, for large, multi-modal public transport networks it can be challenging to summarize the journey records meaningfully. This paper develops a method for categorizing origin-destination (OD) pairs by public transport mode or combination of modes used. By aggregating across OD pairs, this categorization scheme summarizes the multi-modal aspects of public transport network usage. The methodology can also be applied to subsets of data filtered by time of day or geography. The categorization results can inform performance analysis of OD pairs, allowing planners to make comparisons between pairs served by different combinations of modes. London Oyster card data is analyzed to illustrate how the OD pair categorization can characterize a network, allowing planners to quickly assess the roles of different modes, and perform OD pair analysis in a multi-modal public transport network.
\end{abstract}

Keywords Multi-modal · Network Structure - Smart Card · User Behavior . Performance Evaluation · Journey-Based

\section{Introduction}

In public transportation, planning takes place at multiple levels. Pelletier et al (2011) suggest that smart card data can be used at three levels of planning: strategic, tactical, and operational. This paper focuses on using smart card data to aid decision-making

C. Viggiano

Massachusetts Institute of Technology

77 Massachussetts Avenue, Bldg 1-235

Cambridge, MA 02139 Tel.: +1-650-387-8672

E-mail: ceciliav@mit.edu 
at the strategic level, by better understanding the roles of different public transport modes within the network. Do bus services feed rail or are they used as a stand-alone service? Do bus routes provide an alternative to rail for certain trips? The analysis presented here focuses on understanding the use of modes within the public transport network (bus and rail). However, if detailed journey data for other modes, such as car, walk, or cycle are available, the methodology could be extended to categorize OD pairs by these modes as well. The understanding of the role of modes in the public transport network informs the evaluation of the existing network and the identification of opportunities for improvement and expansion.

Several studies have sought to improve understanding of user behavior using smart card data, most commonly with an aim to improve marketing. Utsunomiya et al (2006) combined smart card data with personal information provided by users to analyze typical patterns for different user groups and develop profiles for users of specific stations. Morency et al (2007) analyzed the regularity of daily patterns and classified users based on their public transportation usage. Bagchi and White (2005) analyzed smart card churn to inform targeted campaigns to retain users. These studies can inform decisions about advertizing, promotions, and fare structure.

In contrast, research on network structure has focused primarily on network topology. Garrison and Marble (1964) used measures derived from graph theory to assess connectivity of transportation networks. Other studies built on these measures to characterize network shape (Gordon, 1974), inform network design (Vuchic and Musso, 1991) and identify properties of network structure (Derrible and Kennedy, 2010).

Instead of beginning with network topology, this paper introduces a journeybased approach to explore the multi-modal properties of networks. Extensive usage of smart cards for payment makes this approach possible. Smart cards record passengers' entries and in some cases exits from the public transportation system. Several researchers have developed methodologies to reconstruct individuals' itineraries, inferring origins, destinations, and transfers (Chu and Chapleau 2008; Gordon et al 2013). In networks where smart card usage is prevalent, these itineraries can provide a comprehensive picture of public transit travel.

In large networks such as London, where there are typically upwards of 15 million smart card transactions per day, the data must be aggregated to provide insight. This paper proposes a methodology that consists of two steps: the clustering of stops and stations for grouping journeys into zonal OD pairs, and the categorization of OD pairs based on the mode or combination of modes used. In the context of this research, modes are defined as bus and rail. The categories can be applied to all OD pairs, a subset of OD pairs, or a subset of the journeys for each OD pair, allowing for exploration of temporal and spatial variation. This methodology provides a concise representation of the modal attributes of public transport network usage that can inform planners making decisions about network structure.

The stop and station clustering and OD pair categorization processes can also serve as a foundation for planners seeking to evaluate performance and make comparisons across a multi-modal network. While performance indicators are often calculated at the route or line level, performance at the OD pair level more closely reflects passenger experience and can take into account multiple paths serving the same OD pair. The stop and station clustering methodology defines zonal OD pairs that serve 
as the fundamental unit of analysis for OD level performance evaluation. Given that modes have different properties, planners may wish to take mode into account in assessments of of a multi-modal network. The categorization scheme proposed in this paper can be used to associate OD pairs with the set of modes used.

The methodology is demonstrated using the London public transportation network as a case study. Stops and stations are clustered into zones, and zonal OD pairs are assigned to one of seven modal categories. Time of day and geographic variation in OD pair categorization is presented, and distance and speed profiles are estimated for the most populated categories.

\section{Methodology}

As inputs, the methodology requires data for one-way complete journeys consisting of, at a minimum, initial and final stops or stations and mode or combination of modes used. The stops and stations are clustered based on location to assign journeys to zonal OD pairs according to their initial and final stops or stations. Then OD pairs are categorized based on the share of journeys by each mode or combination of modes.

\subsection{Definition of a Zonal Scheme}

In many cases, individuals consider multiple paths within the public transport network to make their journey. For example, they may have a choice between parallel bus and rail lines, or between neighboring stops on the same route, or they may choose between different paths within the public transport network that follow different trajectories but nonetheless start and end in similar locations. Figure 1 illustrates an example of three paths that serve the same origin and destination. Path 1 uses a bus route that provides direct service from the origin to the destination. Paths 2 and 3 use the same combination of a bus route and a rail line, but start at different origin stops.

To account for multiple paths, such as the three paths in the example, transportation analysis often groups origin and destination points into origin and destination zones. Aggregating journey data into discrete and mutually exclusive zonal pairs allows for journey-based analysis that assesses the existence of multiple paths in the network and can summarize behavior in the entire network without risk of doublecounting journeys. If complete journey data including access and egress stages is available, true origin and destination points (such as the individual's home or office building) can be grouped into zones, allowing for accurate assignment of complete journeys to zonal OD pairs.

In most cases, however, data on the access and egress portion of public transport journeys is much less widely available than within-network journeys data, as it is typically more costly and difficult to collect. This methodology is designed for the analysis of smart card data, which is available in many networks, and typically provides good coverage of travel throughout the network, but does not provide information about true origins and destinations for each journey. Therefore, the methodology 


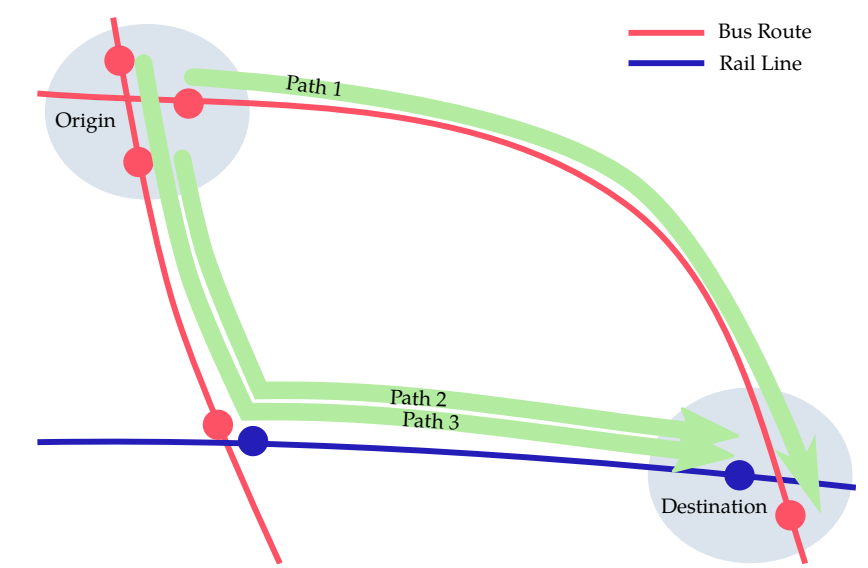

Fig. 1 Example of multiple paths serving a similar origin

assumes that journeys begin (end) in the area surrounding their origin (destination) stop or station. Stops and stations can be grouped into zones with journeys assigned to zonal pairs based on their starting (ending) stop or station. This methodology is reasonable where walking is the main mode to access the public transport system.

The definition of a zonal scheme introduces several challenges. First, there is individual variation in the access distance and egress distance that individuals are willing to include in their journeys. Therefore, the access area around a stop or station cannot be universally defined. Secondly, all zonal schemes present boundary issues. True origin and destination points as well as stops and stations may be located on or close to the boundaries of zones. When this is the case, it becomes more likely that the origin (destination) stop or station is not in the same zone as the true origin (destination).

One way to group nearby stops and stations is to use existing zonal schemes, such as postcodes or census tracts. In these schemes, the zones are typically defined using roads as boundaries. Consequently, bus stops and rail stations tend to be at the boundaries of zones, increasing the likelihood that individuals access the stop or station from a neighboring zone. Figure 2 shows the zonal boundaries of London's postcode sectors as well as those defined by the London Transportation Studies (LTS) model. In the case of the postcode sectors, a large proportion of bus stops and rail stations are located along the borders of zones. This is true for the LTS zones as well, though to a lessor extent.

In networks with dense public transport coverage, it may be impossible to entirely avoid having stops or stations at the boundaries of zones, but zonal schemes can be designed to minimize this issue. Clustering algorithms assign data into groups based on in-group similarity and between-group separation. By defining each stop or station by its geographic coordinates (latitude and longitude or equivalent), clustering algorithms identify distinct clusters of stops or stations located in close proximity. Defining zones using a clustering method, rather than using an existing zonal scheme, can reduce the incidence of stops or stations on or close to zonal boundaries, in turn 

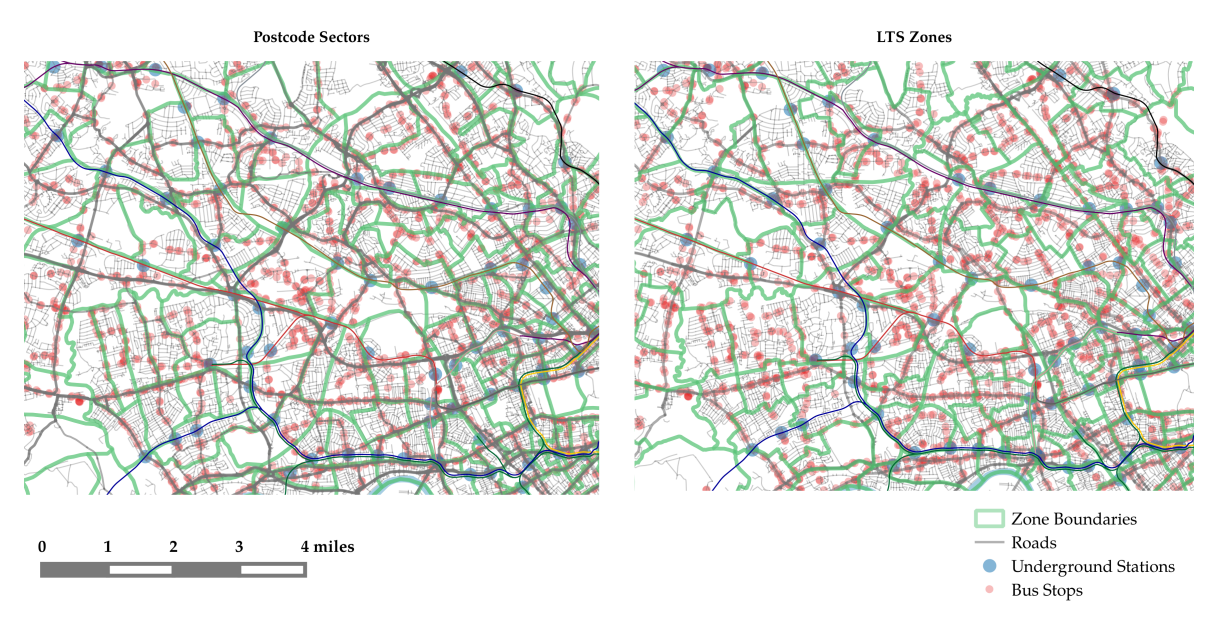

Fig. 2 Examples of existing zonal schemes

reducing the probability that stops or stations are accessed from true origins in neighboring zones.

\subsubsection{Clustering Methodology}

In order to assess the similarity or dissimilarity of data points, all clustering methods use a distance measure to define data closeness. Because data points in this application represent physical points in space, defined by their latitude and longitude, Euclidean distance is an obvious choice of distance measure. Of course, the straight line distance between stops or stations is an approximation of the actual walking distance along streets and pedestrian ways. Using network path distance would be more accurate, however it requires more information - detailed road network data and assumptions about walking paths. Given that true origins and destinations are unknown and zones are therefore intended only as an approximation of origin and destination location, Euclidean distance was deemed an adequate measure of closeness.

There are many methods for clustering data based on feature similarities. These include density-based methods, hierarchical methods, and partitioning methods.

Density-based methods assume that the data consists of a mixture of points drawn from many different probability distributions, with each cluster representing a specific distribution. The algorithms grow initial clusters as long as the density in the neighborhood of the clustered points meets a density threshold. One common densitybased method, DBSCAN (density-based spatial clustering of applications with noise) is efficient for large spatial databases, such as the stop and station location data in this application (Maimon and Rokach, 2005). However, DBSCAN does not assign all data points to clusters. Clusters are only identified where the minimum density threshold is met, with points outside of these clusters left unassigned. This aspect of the methodology is problematic for the application presented here, as all stops and stations must be assigned to a zone for the downstream analysis. 
Hierarchical clustering methods either merge or divide data points into clusters using rules about cluster linkage, which define the distance between clusters. Hierarchical methods are flexible and can identify non-isotropic clusters including chainlike or concentric clusters. However, they have non-linear complexity, meaning they may not be appropriate for clustering large data sets. Hierarchical algorithms output a dendogram, which presents nested clusters representing different levels of similarity. While hierarchical clustering does not require the user to pre-determine the number of clusters, the user must determine which level of the dendogram to use to define clusters (Maimon and Rokach, 2005).

Partitioning methods begin with an initial partitioning and then reassign data points based on feature similarity. They typically require the user to specify the number of clusters. The k-means algorithm is the most used partition method and works well for data that forms isotropic, convex clusters. It is unable to accurately identify non-convex clusters such as concentric circles or clusters that are highly variable in size (Maimon and Rokach, 2005).

While both hierarchical and partitioning methods may be able to produce reasonable clusters from the stop and station location data, the k-means algorithm was selected for several reasons. The k-means algorithm assigns points to clusters based on the distance to the cluster centroid, producing voronoi polygon-shaped zones surrounding each cluster centroid. This method matches the aim of the application: the spatial clustering of coordinates, in which ideal zones consist of a cluster of stops or stations close to the zonal centroid, with a buffer surrounding them. In addition, applying k-means is computationally efficient compared to hierarchical methods (Maimon and Rokach, 2005).

Furthermore, the two most commonly cited weaknesses of k-means, the fact that it is limited to isotropic clusters, and the requirement that users specify the number of clusters, do not pose problems for this application. Non-uniform, concentric, and irregular shaped clusters are not expected nor desired. And while the exact optimal number of clusters is unknown, the approximate number of clusters that is appropriate given information about access and egress distances is known. Section 2.1.2 discusses the selection of the number of zones in more detail. Because zone size is an important consideration in this application, the user specification of the number of clusters gives the user more control over this aspect, compared to some other methods.

The k-means algorithm consists of three steps. First, a set of data points are selected as the initial centroids. The number of centroids corresponds to the userspecified number of clusters. Then, each stop or station is assigned to the closest centroid. In this case, closeness is measured using the Euclidean distance between the coordinates. Once all points have been assigned, the centroids are recalculated as the mean coordinate values of all points assigned to a given cluster. This process iterates until the locations of the centroids do not change significantly from one iteration to the next (Lloyd, 1982).

The algorithm always converges, but may reach a local (instead of a global) minimum, specific to the selection or initial centroids. Therefore, the algorithm is typically run multiple times to find the results with minimum in-cluster variation (MacQueen, 1967). In addition, in order to improve performance, the k-means++ initialization process can be used to select the initial centroids. The k-means ++ initialization process 
ensures that initial centroids are distributed throughout the data set. To achieve this, initial centroids are sequentially sampled from a weighted version of the data set, with weights assigned proportional to the squared distance from the closest initial centroid already selected. The process is fast and improves both the speed and performance of the k-means clustering methodology (Arthur and Vassilvitskii, 2007).

The k-means algorithm clusters data points, assigning equal importance to each point. However, rail stations and bus stops have different properties. Typically, networks have fewer rail stations than bus stops, but each rail station draws a much higher ridership. Positioning high ridership rail stations in the center of zones can reduce violations of the assumption that journeys' true origins (destinations) are in the same zone as origin (destination) stops or stations. Furthermore, given that the analysis focuses on the roles of different modes, generating clusters with rail stations at the center is helpful in ensuring that instances of mode choices are identified. Therefore, it can be advantageous to weight stops and stations by number of boardings prior to applying the k-means algorithm. The impact of stop and station weighting is discussed in Section 3.2.1. A more simplistic option, weighting rail stations by a factor over bus stops, is also discussed, and demonstrated to be more effective.

\subsubsection{Number of Zones}

The number of clusters is a user-specified input to the k-means algorithm. For this particular application, the size of zones is important. If there are too many zones, individuals are likely to consider stops and stations in multiple zones, and the stops and stations in a single cluster will attract users from multiple zones. At the same time, the stops within each cluster should be in comfortable walking distance of one another, as the analysis assumes that paths originating from any stop or station in a zone constitute valid alternatives for travel on zonal pairs. Too few clusters can result in stop and station groupings that violate this assumption.

Given these objectives, information about access and egress distance distributions in a network is important in choosing the appropriate number of zones, and resulting zone size. Additionally, data on complete journeys, including the access and egress portions (if available) can be used to validate the choice of zonal scheme, by assessing the extent to which journeys' true origins (destinations) are in the same zone as their origin (destination) stop or station. The use of access distance and complete journey data in the zone definition process is demonstrated for the London application in Section 3.2.1.

Finally, clustering methods often use various metrics to select the number of clusters. The silhouette score evaluates cluster tightness (the closeness of points within a cluster) and cluster separation (the distance between clusters) (Rousseeuw, 1987). Cluster tightness, denoted by $a(i)$ is the average distance from a point to all other points in the same cluster. Cluster separation, $b(i)$, is measured as the average distance from the point to all points in the closest neighboring cluster (based on the distance between point $i$ and all other cluster centroids). The score for each point is calculated as:

$$
s(i)=\frac{b(i)-a(i)}{\max \{a(i), b(i)\}}
$$


The resulting score is between -1 and 1 . Scores that are lower than 0 indicate that the point is not well-clustered, and is a better fit with another cluster. A high score suggests the point is well-clustered in that it is much closer to other points in its own cluster than to the closest neighboring cluster. The silhouette score can be aggregated to the cluster level by taking the average $s(i)$ for all points in a cluster, or it can be aggregated to the entire data set by taking the average $s(i)$ for all points. The score for the full data set can be used to compare different clustering results, and to select the appropriate number of clusters. The best choice is considered to be the number of clusters that produces the largest overall average silhouette score (Rousseeuw, 1987).

\subsection{Categorization of Origin-Destination Pairs}

The result of the stop and station clustering is a set of zones with each stop and station belonging to a single zone. Given data on complete one-way journeys taking place in the network, these journeys can be assigned to zonal OD pairs according to the zones of their initial and final stop or station. These complete journeys are also classified by mode, defined as either bus (all stages were by bus), rail (all stages were by rail) or combined (journeys including both bus and rail stages). As a result, each zonal OD pair is defined by three variables: percent bus, percent rail, and percent combined. The categorization method assigns OD pairs to categories based on these percentages.

Section 2.2.1 identifies seven distinct combinations of these modes and presents a method to assign OD pairs to the seven categories using user-defined thresholds. An alternative method for defining categories, discussed in Section 2.2.2 is to use a clustering methodology to identify groups of OD pairs with similarities across the three variables (percent rail, percent bus, percent combined).

\subsubsection{User Defined Categories}

Given that all journeys are defined as either bus, rail, or combined, the following is an extrapolation of all possible combinations of these journey types.

- primarily bus

- primarily rail

- primarily combined

- bus and rail

- bus and combined

- rail and combined

- bus, rail and combined

To assign OD pairs to categories, we define a dominance threshold and an existence threshold. The dominance threshold is the percentage of journeys by a given mode required to place the OD pair into the single-mode categories (primarily rail, primarily bus, and primarily combined). If the dominance threshold is $80 \%$, OD pairs with $80 \%$ (or more) journeys by rail will fall into the primarily rail category, and likewise for primarily bus and primarily combined.

The existence threshold is the minimum percentage of journeys by a certain mode to include the mode in the category assignment. If the existence threshold is $10 \%$, 
an OD pair with $5 \%$ rail, $65 \%$ bus and $30 \%$ combined would fall into the bus and combined category and not the bus, rail, and combined category.

The assignment is rule-based, using the dominance and existence threshold in a two-phase process. First, any pair in which the journeys by one mode exceed the dominance threshold is assigned to the appropriate dominant mode category (primarily rail, primarily bus, or primarily combined), regardless of the percentage of journeys by the other modes.

If no mode exceeds the dominance threshold, the pair is assigned to a category based on which of the three modes exceed the existence threshold. If all three exceed the existence threshold, the pair is assigned to the 3-mode category (bus, rail, and combined). If only two modes exceed the threshold, the pair is assigned to the appropriate 2-mode category.

Given three possible modes, as in the London case study, the existence threshold $\left(E_{T}\right)$ as a function of the dominance threshold $\left(D_{T}\right)$ must always satisfy:

$$
E_{T} \leq 0.5 *\left(1-D_{T}\right)
$$

to avoid cases where only one mode exceeds the existence threshold. Therefore, each zonal pair is assigned to exactly one category.

Figure 3 visualizes the seven category definitions with the thresholds and example modal percentages plotted to the left of the schematic showing paths between zones.

One challenge of this categorization method is determining appropriate existence and dominance thresholds. Analysis of the distributions of the percent of journeys of a given type across all zonal OD pairs can inform the threshold choice. In addition, planners may wish to vary thresholds for specific applications. A very high dominance threshold and low existence threshold will identify the OD pairs where users appear to be truly captive to a given mode. However, this will result in a high degree of variation in modal splits for the multi-modal categories. If planners wish to identify OD pairs with even modal splits, the existence threshold can be raised and the dominance threshold lowered.

\subsubsection{Unsupervised Category Identification}

A more exploratory method for category definition is to assign zonal OD pairs to categories using a clustering method, with zonal pairs clustered based on their percent rail, percent bus, and percent combined attributes. Either the hierarchical or partitionbased methods discussed in Section 2.1.1 could be used to identify modal categories.

The clustering methods identify groups of OD pairs with similar mode shares. This method does not require the user to specify thresholds or even the modal makeup of each category. However, a partition method like k-means requires the user to specify the number of clusters, and hierarchical methods require the selection of the level of the dendogram at which clusters are defined.

The benefit of using a clustering method to define OD pair categories is that it can help identify and define categories that users may not have considered. However, these categories may be more difficult to understand and communicate due to the lack of defining thresholds. In addition, some networks may not have clear cluster separation on these variables. 

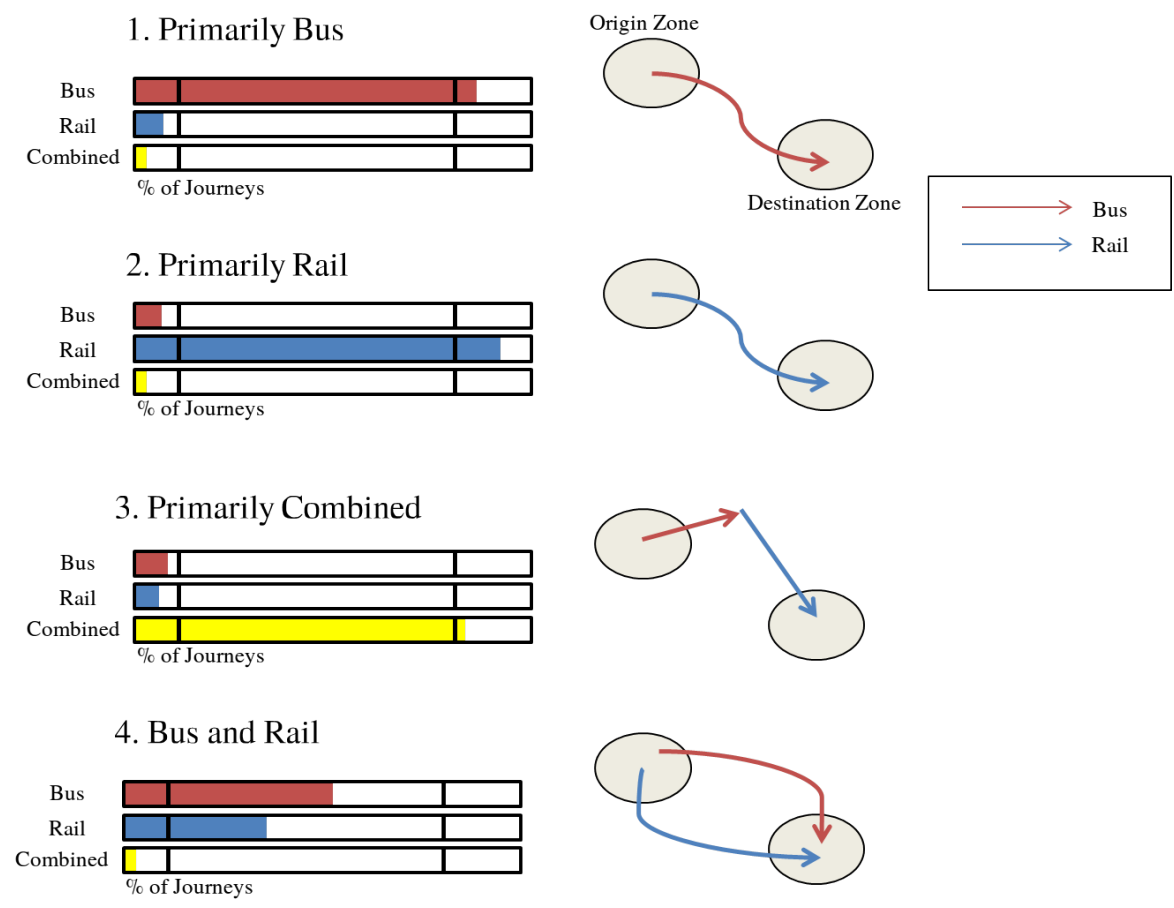

5. Bus and Combined
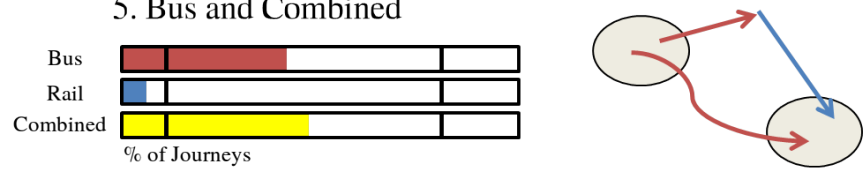

6. Rail and Combined
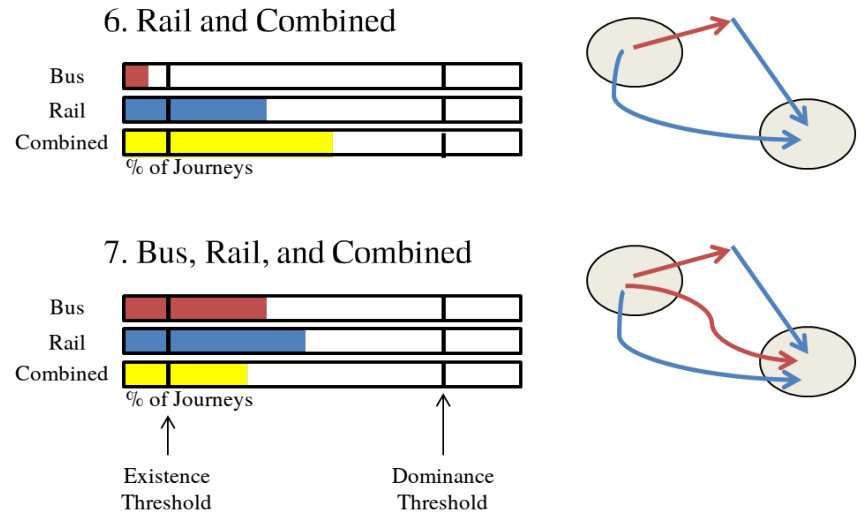

Fig. 3 Seven modal categories 


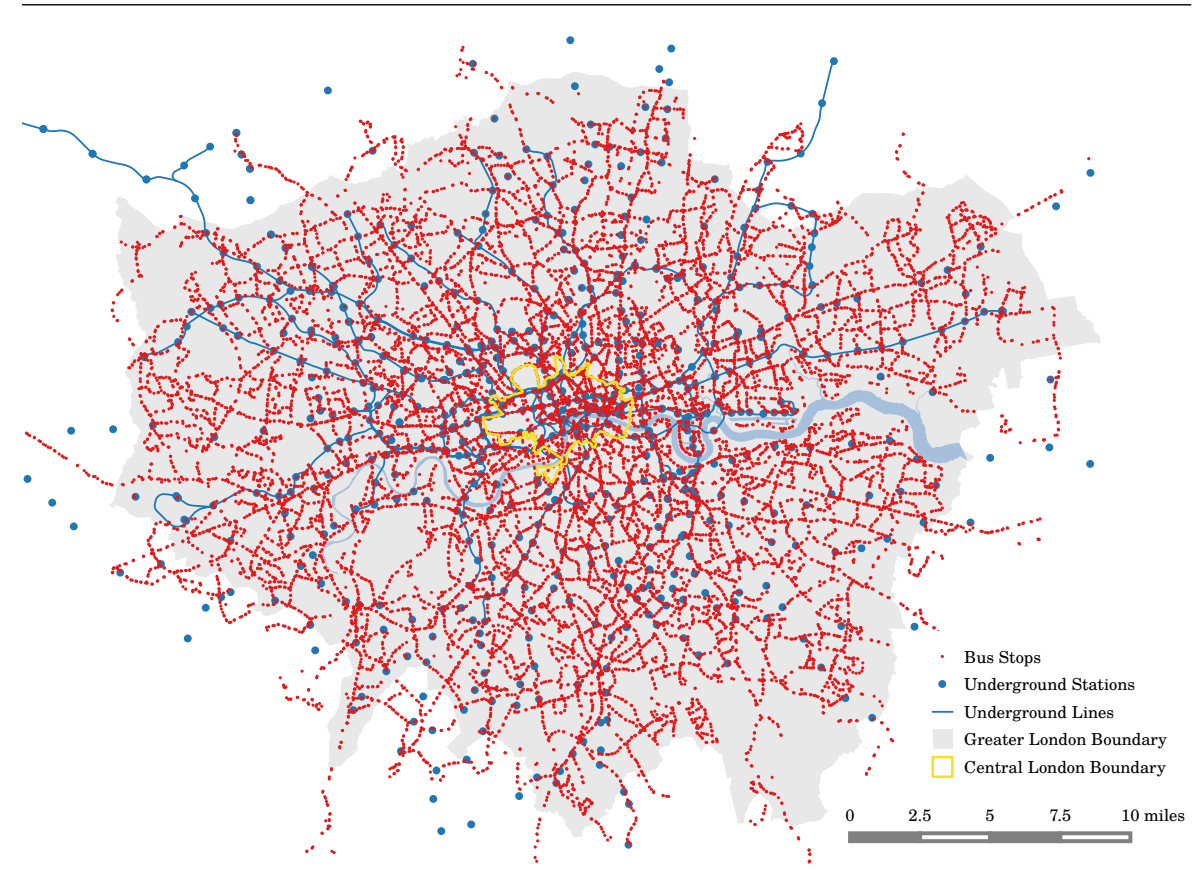

Fig. 4 Map of London rail stations and bus stops

\section{London Case Study}

London provides an interesting example for this analysis because it has a multi-modal public transportation network and a high rate of population growth necessitating growth in the bus system. As planners make decisions about how to accommodate increased demand for bus service, they must first understand the role of bus in the current network. Figure 4 shows a map of London's multi-modal network.

\subsection{Oyster Data Set}

The smart card data used for the case study consists of 14 days of Oyster (London's smart card) transactions. The data was processed using ODX, a methodology developed by Gordon et al (2013) which infers origin bus stops, alighting bus stops, and links stages of multi-stage journeys using automatic vehicle location data and geographic and time-based thresholds. For simplification, only journeys of up to three stages were included (with stages here defined as initiating with a smart card tap), which represent $99.4 \%$ of all journeys.

Figure 5 displays the distribution of journeys by mode in London for the data analyzed. Underground, Overground, and National Rail are grouped as rail for this analysis. In the Underground, passengers can transfer between lines without tapping their card during the transfer. Therefore, we do not always know the exact path an 


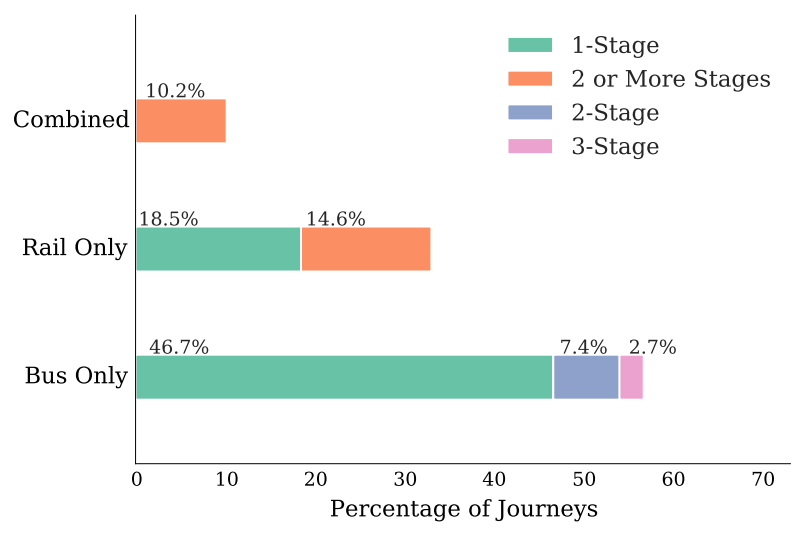

Fig. 5 Journeys by mode and number of stages

individual takes through the rail network. If an individual's starting and ending station are served by the same line (i.e. the Victoria line), it is assumed to be a single rail stage. Otherwise the number of stages is designated as two or more. Passengers must tap their Oyster card at the beginning of each bus stage so bus journeys can be accurately defined as one, two, or three stages.

The ODX methodology inferred the starting and ending stop or station for $81 \%$ of journeys made using an Oyster card in the two weeks analyzed. Of the Oyster journeys that did not have a starting and ending stop or station inferred, $69 \%$ were identified as single stage bus journeys with an origin stop and bus route but no destination stop inferred. This is because in the London network, passengers must "tap out" of the rail network, but they do not "tap off" of bus journeys. The ODX methodology infers the alighting stop for bus journeys based on the next stage of the journey or the same day return journey, meaning that destination stops for bus stages without a continuation or return journey cannot be inferred.

Because the proposed analysis is OD-based, journeys without destination stops cannot be included, meaning that the disproportionate inference rate for single stage bus journeys would result in under-representation of bus journeys. To correct for this under-counting, each of the single stage bus journeys with an uninferred destination stop was assigned an alighting stop by the following methodology: For each boarding stop, a destination stop distribution was constructed, consisting of the frequency of occurrence of all inferred downstream destination stops for single stage bus journeys originating at that stop. Then, for each journey beginning at that boarding stop which did not have an inferred destination, a destination stop was selected at random from this distribution. This methodology assumes that single stage journeys with uninferred destinations have the same destination distribution as single stage journeys with inferred destinations.

Through the inference methodologies, origin and destination stops or stations are inferred for $94 \%$ of journeys paid for with an Oyster card, or 90,306,224 journeys in the 10-day period. Assuming Oyster cards were used to pay for approximately $80 \%$ 
of journeys in the period (Transport for London, 2012), this data represents 75\% of all journeys in the period.

\subsection{London Stop and Station Clustering}

As discussed in Section 2.1.1, the k-means algorithm was selected for the clustering of stops and stations into zones. The following section presents the analysis performed to determine the appropriate stop and station weighting and number of zones for the application of k-means.

\subsubsection{Stop and Station Weighting and Selection of ' $k$ '}

Given the importance of zone size as it relates to access and egress distance, the first consideration is the approximate zone size resulting from different choices of ' $\mathrm{k}$ '. Table 1 shows the resulting average zone sizes for 750, 1,000, and 1,250 zones. The table also presents the approximate maximum distance to traverse a zone: this is calculated as the diagonal distance distance across the zone, assuming equal-sized, square zones.

\begin{tabular}{lccc} 
Number of Zones & 750 & 1,000 & 1,250 \\
\hline Average zone size (square miles) & 0.82 & 0.62 & 0.49 \\
Approximate maximum zonal traverse distance (miles) & 1.28 & 1.11 & 0.99
\end{tabular}

Table 1 Average zone size by number of zones

Data from the London Travel Demand Survey (LTDS), an in-person household level travel diary survey suggests the average straight line distance from a journey's origin to the boarding bus stop or rail station is approximately 0.42 miles. Furthermore, $75 \%$ of access stages for public transport journeys are less that 0.6 miles and 93\% are less than 1 mile, measured as straight line distance.

Given this information about access distances in London, at least 750 zones should be defined. For 750 zones, the average maximum distance to traverse a zone is 1.28 miles, which is approximately the 97th percentile of access distances recorded by LTDS. While most individuals will not have to traverse the entirety of the zone to reach a stop or station, having fewer than 750 zones is likely to produce zones that include walking access distances that many individuals are unwilling to undertake. Defining more than 1,250 zones is also unreasonable. With 1,250 zones, the diagonal distance across the zone is 0.99 miles. This means that the average access distance ( 0.42 miles) observed in LTDS from the center of the zone would extend nearly to the edge of the zone. If more than 1,250 zones are defined, it will become very likely that individuals access stops or stations in a given zone from neighboring zones. Therefore, the range of 750 to 1,250 zones was considered.

In order to select the number of zones from this range, and also to decide whether to apply a weighting scheme, we use information about true origins inferred from 
Oyster card registration data. In London, Oyster card registration includes the provision of a home postcode. Assuming that the first journey of the day began at this home address, the registration postcode can be linked to the Oyster card journeys analyzed to infer the true origin location for these journeys. Overall, the access distance distributions inferred using this linkage methodology were found to match well with data from the London Travel Demand Survey (Viggiano et al, 2016). Not all Oyster cards are registered, and some registration postcodes were unrealistically far from the initial boarding stop or station, suggesting the individual either moved or did not begin the first journey from home. Using postcodes within 1 mile of the boarding bus stop and within 1.25 miles of the boarding rail station, true origins can be inferred for 343,775 journeys. This sample is used to evaluate clustering alternatives.

For evaluation purposes, the percent of these journeys for which the zone assigned to the boarding stop or station matches the zone assigned to the postcode (postcodes in London are small, representing on average 15 properties and are geocoded to a single point) is calculated. Three different weighting methodologies are considered. In the first case (unweighted), each stop and station is treated equally. However, as discussed in Section 2.1 it may be beneficial to ensure that high ridership stops and stations are in the center of clusters. Weighting stops and stations by boardings will draw zonal centroids closer to high ridership stops and stations, ensuring they are more centrally located within each zone. For the second case (journey-weighted), stops and stations are weighted by number of boardings. The third case is a simplification of the second case. Instead of weighting each stop and station by ridership, a flat weight of 10 is applied to all rail stations (rail-weighted) to reflect the fact that rail stations have much higher ridership than bus stops.

Figure 6 shows the percent of journeys with postcodes assigned to the same zone as the boarding stop or station for the three weighting methodologies with 750, 1,000, and 1,250 clusters. The matching rate is expected to decline as the number of clusters increases, due to the resulting decline in zone size. The rail-weighted method consistently provides the highest rate of stop and postcode matching. While the differences are small, a t-test reveals that the differences between the rail-weighted and unweighted methods are statistically significant. Furthermore, the differences between the rail-weighted and journey-weighted are also statistically significant. The fact that the rail-weighted method outperforms the journey-weighted method is somewhat unintuitive, as it would be expected that the journey-weighted method would ensure that high-ridership stops and stations are in the center of zones, increasing the chance that they are accessed from postcodes within the zone. However, given the wide variation in boardings per stop and station, the journey-weighted method results in geographically small clusters surrounding high ridership stations which reduces the chance of the postcode matching the boarding stop or station zone. Given that the rail-weighted method is simple to apply and produced the best results, the rail-weighted method was selected.

The matching percentage will tend to decline as the zone size decreases, therefore this metric is not useful for selecting the number of zones. Instead, the overall average silhouette score, as discussed in Section 2.1.2 is estimated for 750 through 1,250 clusters. Figure 7 shows the results. For the silhouette score, a peak indicates 


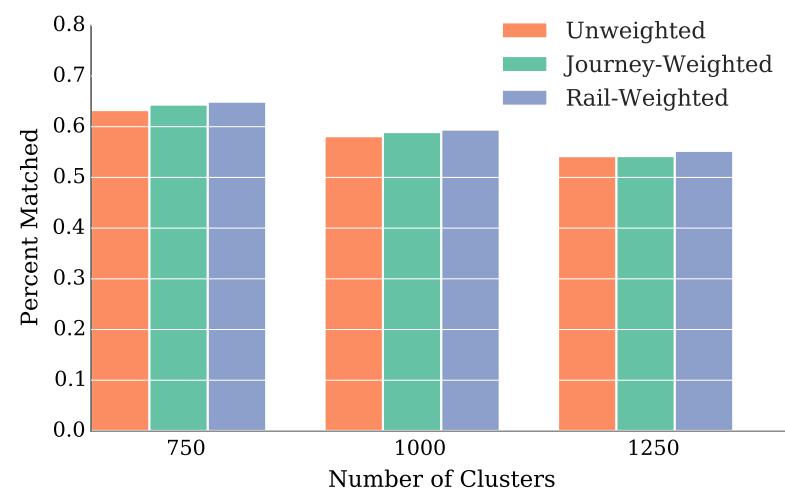

Fig. 6 Postcode and stop (station) match rate by weighting method

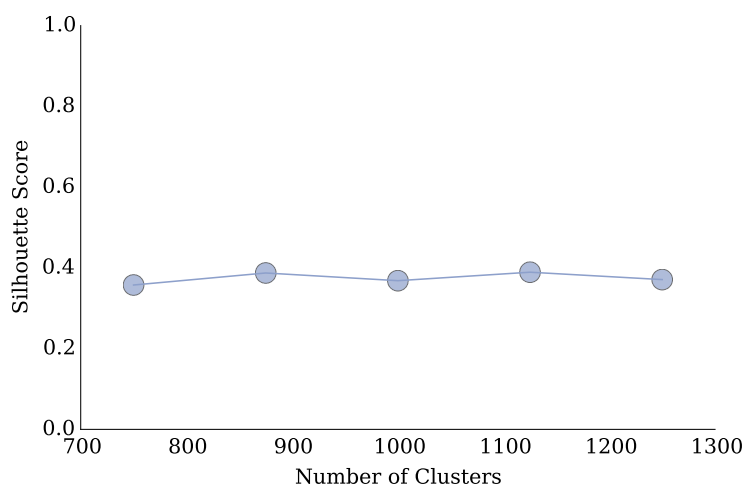

Fig. 7 Silhouette scores for different numbers of clusters

the optimal clustering. However, for the stop and station data, the silhouette score remains flat for 750 through 1,250 zones.

The lack of insight from the metric suggests that the stop and station data may not have a strong underlying cluster form. This is not unexpected, as public transport planners typically design networks stops somewhat evenly spaced to provide good coverage. The London network has dense, even coverage and while there are clusters of stops and stations at activity centers and intersections, not all stops and stations belong to a clear cluster. Despite this, the clustering technique helps reduce the issue of having stops and stations at the boundaries of zones, discussed in Section 2.1.1, relative to existing zonal schemes. For example, the London Transportation Studies (LTS) model defines 949 zones in Greater London. Using the linked postcode and boarding stop (station) data the percent of postcodes located in the same zone as the boarding stop is just $52.8 \%$. This significantly under-performs all of the k-means 


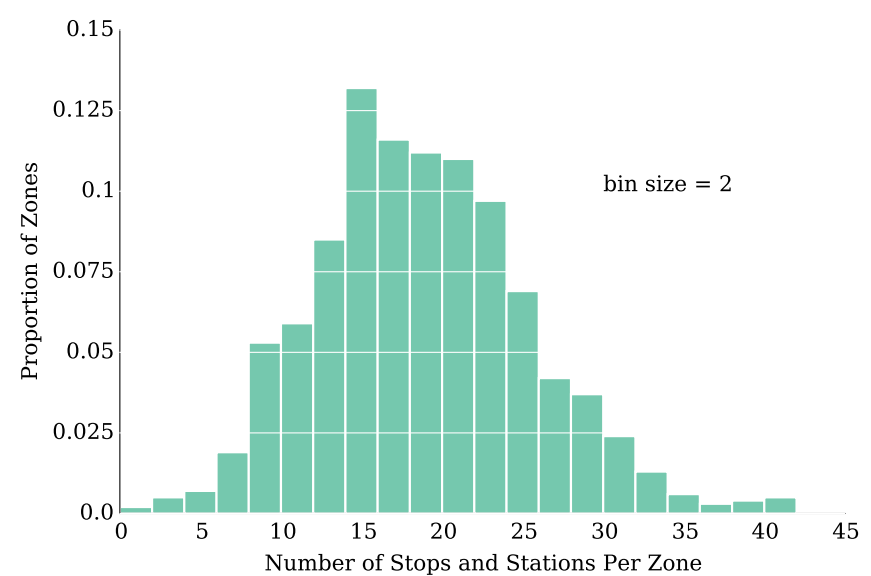

Fig. 8 Distribution of rail stations and bus stops per zone

clustering results presented in Figure 6. The match rate for 1,000 zones using the rail-weighted method is $59.4 \%$.

Ultimately, given the lack of insight from the silhouette score, the number of clusters was selected to produce zones that match the zone size dictated by the access distance distributions. At the center of the range of values considered, 1,000 zones produces zones in which the maximum distance to traverse the zone is expected to be approximately 1.11 miles. This corresponds to the 95 th percentile of access distances observed in the LTDS data. This was judged to be the most reasonable zones size. And the results of the rail-weighted k-means clustering with 1,000 zones were used for the subsequent analysis.

\subsubsection{Stop and Station Clustering Results}

Because stops and stations are more heavily concentrated in Central London, zones are smaller at the center and larger at the periphery. The number of stops and stations per zone varies as well, as shown in Figure 8. Figure 9 displays the zones generated for a portion of Central London. Compared to Figure 2, it is clear that fewer rail stations and bus stops are at the center of zones. Figure 10 shows all 1,000 zones.

Journeys are assigned to zones according to their initial and final stop or station. As expected, the zonal OD matrix is sparse; $48 \%$ of the OD pairs are empty.

\subsection{London Origin-Destination Pair Categorization}

Section 2.2 discussed two possible methods for classifying OD pairs based on modes. Each method was applied to the London data. Only OD pairs with at least 385 journeys were included to avoid small sample size problems. This is the sample size 


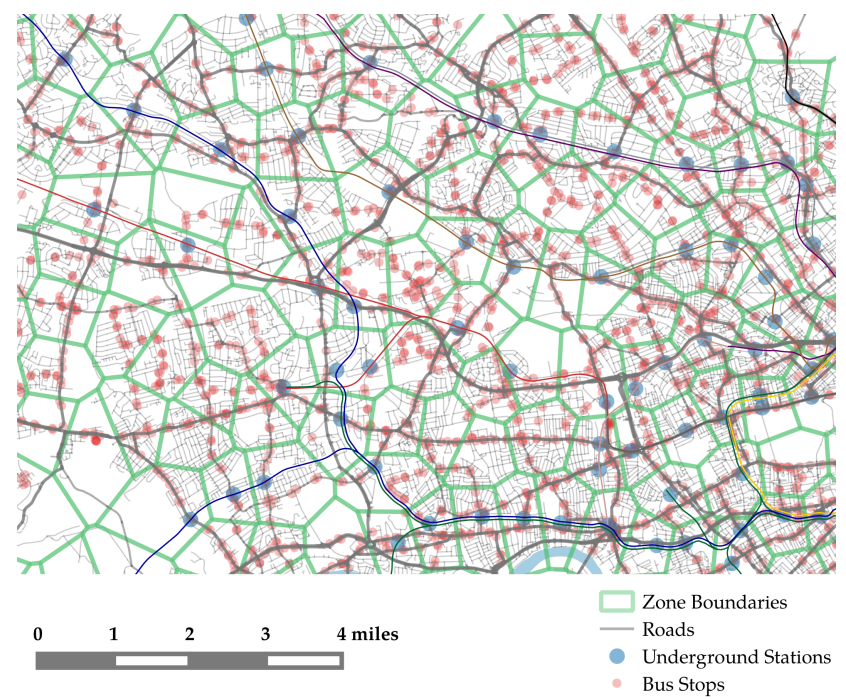

Fig. 9 Central London stop and station clusters



Fig. 10 Map of all zones displaying total AM peak boardings per zone 

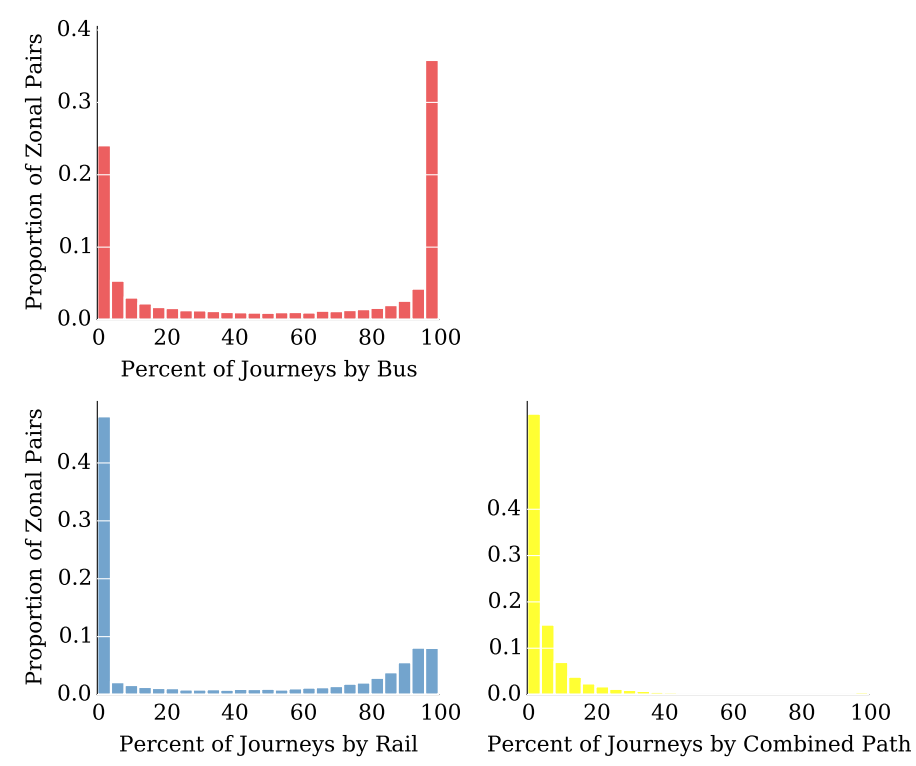

Fig. 11 Distributions of mode share across OD pairs

required to estimate the proportion of journeys by mode with a 5\% margin of error at a $95 \%$ confidence level, assuming no knowledge of the population proportion.

\subsubsection{Categorization Results With User-Defined Thresholds}

For the methodology outlined in Section 2.2.1, the existence and dominance thresholds must be defined. Figure 11 shows the distributions of the percent of journeys by each mode across all analyzed OD pairs. These distributions can inform the selection of the dominance and existence threshold. All three distributions exhibit strong peaking. Therefore the sensitivity to variation of dominance thresholds up to approximately $85 \%$ is minimal, as is the sensitivity to variation of existence thresholds of more than $10 \%$.

For this application, the dominance threshold was a set at $80 \%$. Therefore, according to Equation 2, the existence threshold must be at most $10 \%$. It was set at $10 \%$. This will identify more multi-modal pairs compared to a lower dominance and higher existence threshold.

Figure 12 shows the results for all day as well as the AM peak. In $46 \%$ of OD pairs, bus is the primary mode. This may reflect the fact that many parts of the outer London network are served only by bus. Alternatively, users may have a strong preference for bus for certain journeys, for example short journeys. This comparison between all day and AM peak data, as well as data from other time periods can help planners understand how network usage changes over the course of the day. In Lon- 


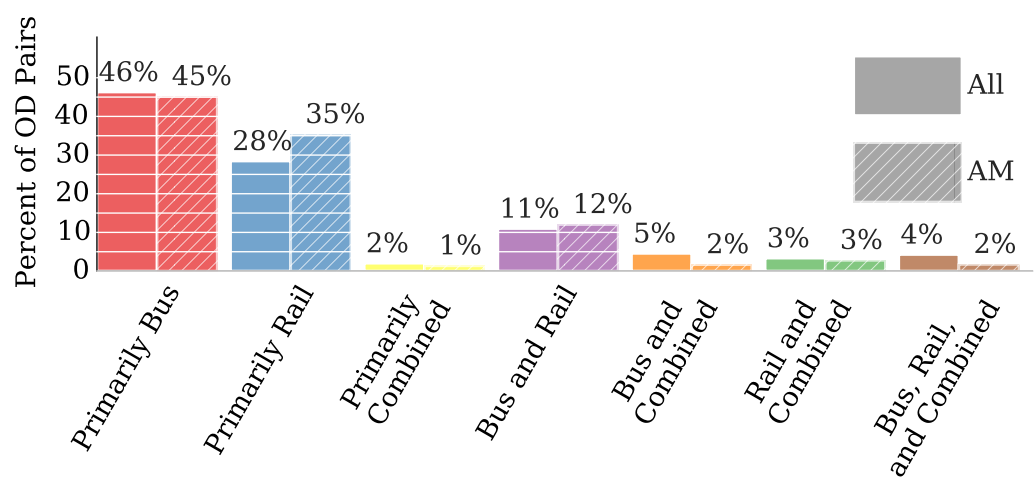

Fig. 12 Categorization of all OD pairs, using all day and AM peak data

don, the percentage of primarily rail OD pairs is greater in the AM peak than overall.

One can also consider geographic variation. To illustrate this, a central region was defined and the following set of journey types were analyzed: OD pairs within the central region (central), OD pairs that start outside the central region and end inside it (to center), OD pairs that start inside the central region and end outside it (from center), and OD pairs that start and end outside the central region (periphery). Figure 13 shows how the categorization results differ between these journey types in the AM peak.

Central OD pairs include a high percentage of bus and rail designated pairs, indicating that both modes are important, in many cases providing parallel service. There is considerable asymmetry in journeys to and from the central zone. The primarily rail category dominates the "to center" OD pairs suggesting that rail is critical for these journeys. However, for the "from center" OD pairs, primarily bus dominates. This is likely due to the fact that destinations outside Central London are less likely to be close to rail stations. Even in the AM peak period, bus service is important for these reverse commuting trips. Not surprisingly, the bus network serves the peripheral trips almost exclusively.

\subsubsection{Results of Unsupervised Category Identification}

Zonal OD pairs were clustered on three variables: percent rail, percent bus, and percent combined. A measure of cluster tightness and separation, such as the silhouette score discussed in Section 2.1.2 can be used to help select the appropriate number of clusters. Figure 14 shows the average silhouette score for three through fourteen clusters. The highest silhouette score is for three clusters, but there is a secondary peak at seven clusters, suggesting that the seven-cluster categorization yields good cluster separation. 
Central

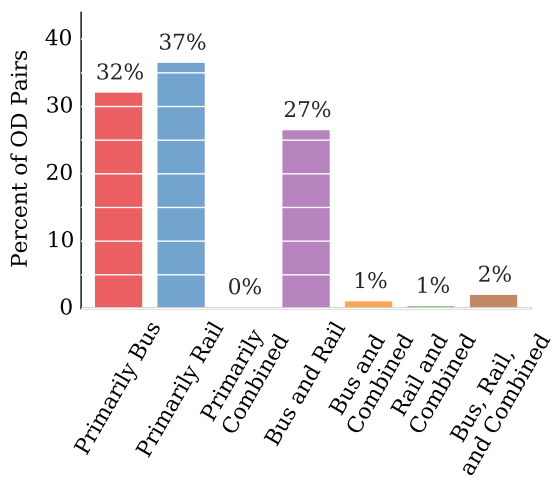

From Center

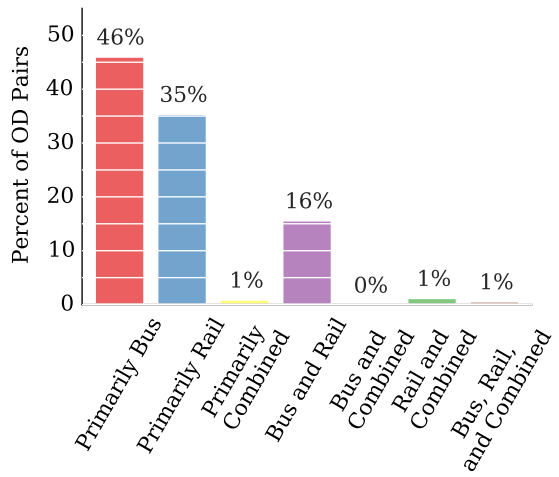

To Center

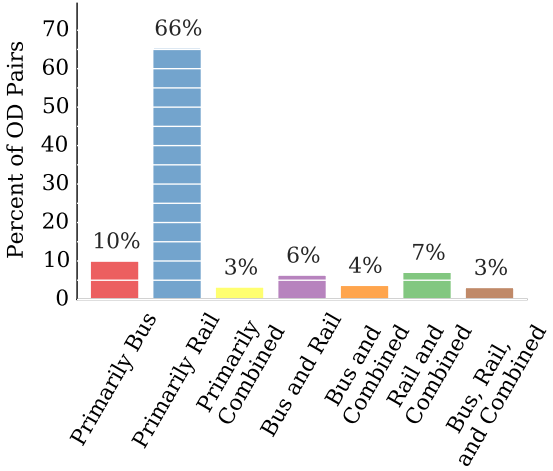

Periphery

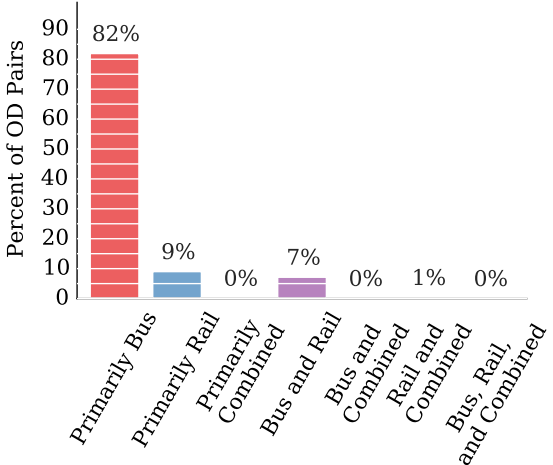

Fig. 13 Geographic variation in categorization results

Using a larger number of clusters identifies more distinct types of zonal OD pairs. Given the peak in silhouette score at seven clusters, the seven-cluster categorization is explored as an example. Figure 15 shows the average percentages for each cluster. Some clusters are difficult to name. Both the "BUS and rail" and "RAIL and bus" clusters have significant bus and rail percentages; the first has more bus and the second more rail. Here, capitalization is used to show this emphasis. While this distinction is interesting, it adds complexity to the presentation of the modal attributes of a network that may be appropriate for some analysis, but confusing for others. An alternative would be to use the clustering for initial categorization, and then make manual adjustments. For example, the "BUS and rail" and "RAIL and bus" categories could be combined, if this distinction is not interesting.

The zonal pair assignment process is also less intuitive compared to the method described in Section 2.2.1. In the user-defined methodology, explicit rules define which category each OD pair is assigned to. When a clustering method is used, the 


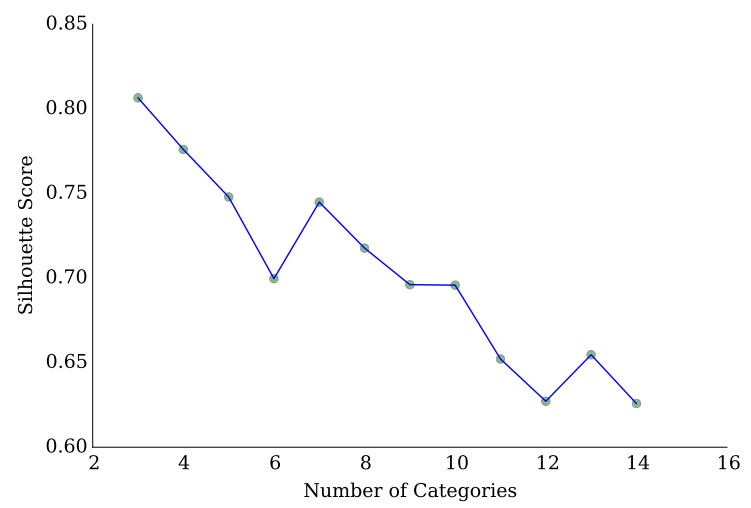

Fig. 14 Overall average silhouette scores for three through fourteen categories

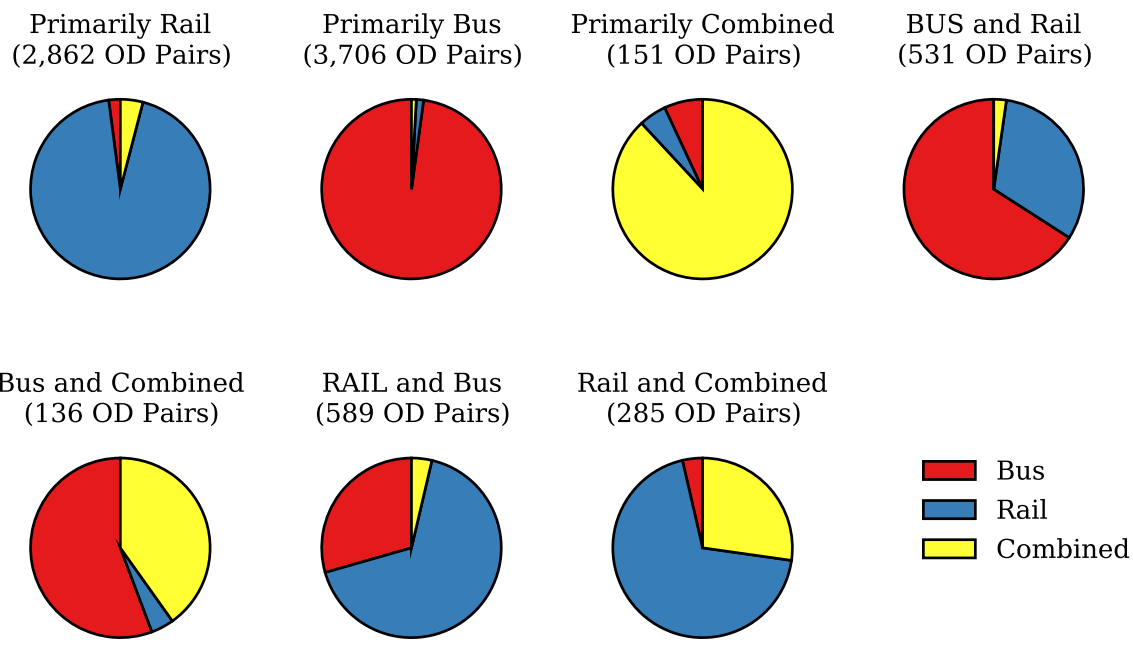

Fig. 15 Average percentages for the seven-category clustering

cluster centroids define each category, and assignment is based on whichever centroid is closest (based on Euclidean distance in three dimensional space, with points defined by the three percentage variables). As a result, there is variation and in the range of percentages assigned to each category. For example, pairs assigned to the "primarily rail" category all have at least $78 \%$ rail journeys while pairs assigned to the "primarily combined" category may have as little as $58 \%$ combined journeys. This reflects the fact that there are fewer combined journeys overall. An argument can be 
made that this variation in delineation makes sense, however, it adds complexity to the category definitions.

Furthermore, the range of values for a given mode assigned to each category have considerable overlap. For example, pairs with up to $31 \%$ bus journeys are assigned to the "primarily combined" category, while the "RAIL and bus" category contains pairs with $13 \%$ to $49 \%$ bus journeys. The overlap is due to the impact of the other modes on the distance to the seven cluster centroids. While this is mathematically straight-forward, it cannot be as easily translated into simple and visual rules as the user-defined categories.

\subsection{Origin-Destination Pair Characteristics and Performance by Category}

Planners can also use the categorization results to assess attributes of OD pairs from each category. These can be descriptive characteristics, such as the distance distribution or evaluation metrics, such as travel speed. For this analysis, selected categories from the "user-defined" method were used, because the categories are intuitive and defined in such a way that is easy to communicate. The analysis focuses on the primarily bus, primarily rail, and bus-and-rail categories. Other categories were excluded from the figure because they make up a small percentage of AM peak OD pairs.

Figure 16 shows the distance distributions for OD pairs that are primarily bus, primarily rail, and bus-and-rail for the AM peak. Distance is defined as the straight line distance from the centroid of the origin zone to the centroid of the destination zone. This shows that in London rail tends to serve longer journeys from 2 to 8 miles while bus serves shorter journeys that range from 0 to 3 miles. The bus-and-rail OD pairs, in which some journeys are made by bus and others by rail, have a distance distribution falling between that of primarily rail and primarily bus, though it more closely mirrors the primarily bus distribution.

Figure 17 plots journey distance against journey time for a random sample of OD pairs, demonstrating the variation in speed across OD pairs. Again, only primarily bus, primarily rail, and bus-and-rail are shown because they are the dominant categories in the AM peak. Primarily bus OD pairs tend to have the slowest speeds but also have less variation in speed compared to primarily rail OD pairs. For OD pair level evaluation, planners can set different standards depending on the category that a particular OD pair is part of.

Planners in London can use the information about distance and speed distributions by category to inform strategic planning decisions. For example, planners may note the overlap in the 1 to 3 mile range between OD pairs in the primarily bus, bus-andrail, and primarily rail categories and determine that they want to implement policies to shift journeys on these pairs from rail to bus. They may also monitor the difference in speed between primarily bus pairs and primarily rail pairs over time to determine if the relative performance changes. They may also use the data to inform decisions about investment in each mode to improve speed. 


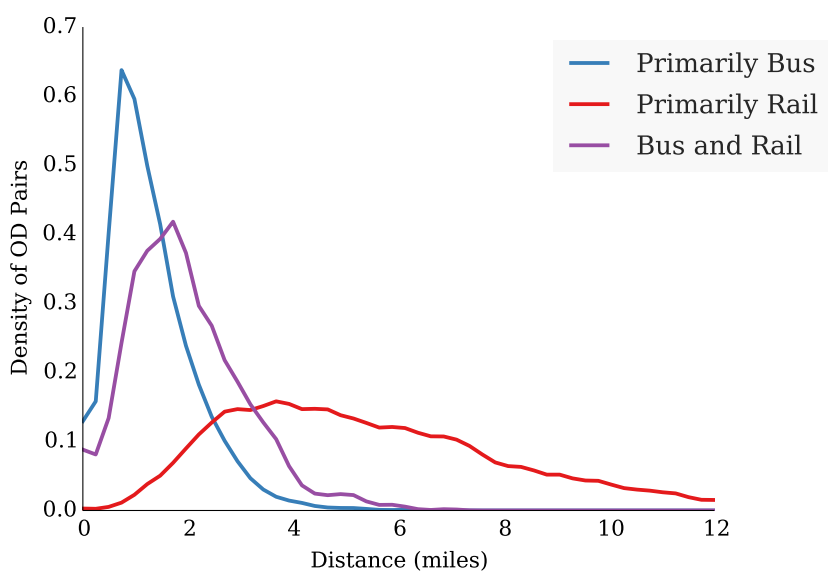

Fig. 16 Distance distributions of OD pairs by category

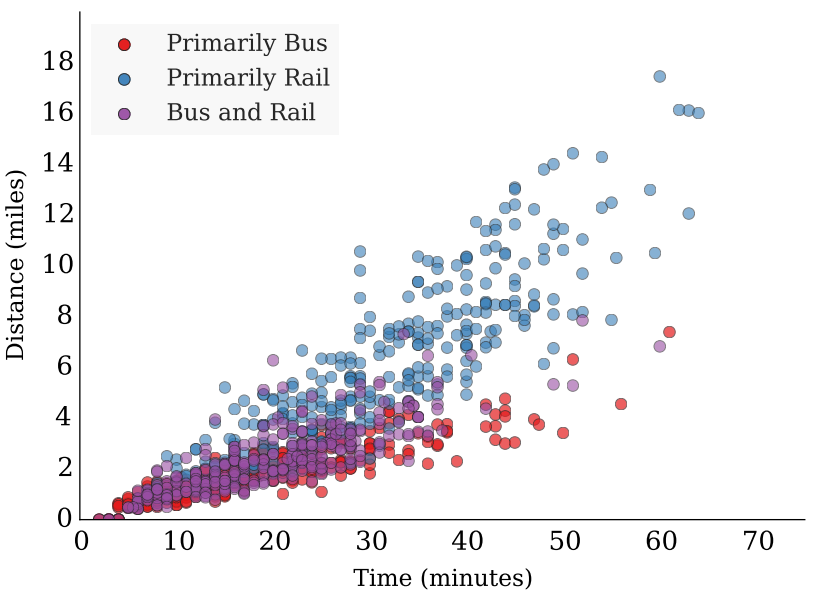

Fig. 17 OD pair distance versus travel time by category

\section{Conclusions}

This paper presents a methodology for aggregating large quantities of smart card data in a meaningful way to help understand the ways in which passengers use different modes in a multi-modal public transportation network. First, the use of clustering algorithms to generate a zonal scheme that positions stops and stations at the center of zones is demonstrated. This enables OD-level analysis of smart card journey data. Then, rather than characterizing the multi-modal characteristics of the network based 
on the network topology, this methodology starts from the passengers' journeys, and presents a clear picture of how the network is used, rather than of the services provided. This understanding of user behavior can inform planners as they make strategic decisions about network structure and modal expansion.

The analysis presented for the London network suggests that bus serves an important role for journeys around the periphery, journeys from the central zone to the periphery, and journeys within the central zone. With this information, planners can decide if they want to focus on improving the services that already fill these roles or concentrate on expanding the role of bus in journeys from the periphery to the center.

The categorization methodology also provides a foundation for further evaluation of multi-modal public transportation networks. Performance metrics assessed at the OD pair level can be useful, particularly in a dense network where passengers may take different paths between an OD pair. A metric that assesses an OD pair based on all public transit paths used provides a better description of service than modespecific evaluations. However, different modes have different properties. In the London application, zonal pairs served primarily by bus tend to cover shorter distances at lower speeds. Using the categories outlined in this research, planners can perform targeted analysis on OD pairs in each category to identify problems and opportunities. Given the differences in the cost and flexibility of bus and rail, as well as difference in users of the two modes, targeted analysis for a specific category may be more effective than more general OD-level analysis. Planners can develop category-specific standards and metrics to account for differences intrinsic to different modes.

Furthermore, the categorization results can help planners understand user preferences and behavior changes over time. For example, planners can analyze the response to fare changes or new services in terms of the proportion of zonal pairs in each category. Or they may observe changes in category membership in the absence of service or fare changes that are the result of external factors such demographic or land use changes. The definition of a zonal scheme and an OD-level categorization methodology allow planners to view the modal attributes of smart card data at a high level to conduct a variety of strategic analyses.

Acknowledgements Many thanks to Transport for London for the support, guidance, and oversight of this research.

\section{References}

Arthur D, Vassilvitskii S (2007) k-means++: The advantages of careful seeding. Proceedings of the eighteenth annual ACM-SIAM symposium on Discrete algorithms pp 1027-1035

Bagchi M, White P (2005) The potential of public transport smart card data. Transport Policy 12(5):464-474

Chu AKK, Chapleau R (2008) Enriching archived smart card transaction data for transit demand modeling. Transportation Research Record: Journal of the Transportation Research Board 2063(1):63-72 
Derrible S, Kennedy C (2010) Characterizing metro networks: state, form, and structure. Transportation 37(2):275-297

Garrison WL, Marble DF (1964) Factor-analytic study of the connectivity of a transportation network. Papers in Regional Science 12(1):231-238

Gordon JB, Koutsopoulos HN, Wilson NH, Attanucci JP (2013) Automated inference of linked transit journeys in london using fare-transaction and vehicle location data. Transportation Research Record: Journal of the Transportation Research Board 2343(1):17-24

Gordon SR (1974) Relationships between economies of scale and the shape of transportation networks. PhD thesis, Massachusetts Institute of Technology

Lloyd S (1982) Least squares quantization in pcm. Information Theory, IEEE Transactions on 28(2):129-137

MacQueen J (1967) Some methods for classification and analysis of multivariate observations. In: Proceedings of the fifth Berkeley symposium on mathematical statistics and probability, Oakland, CA, USA., vol 1, pp 281-297

Maimon O, Rokach L (2005) Data mining and knowledge discovery handbook, vol 2. Springer

Morency C, Trepanier M, Agard B (2007) Measuring transit use variability with smart-card data. Transport Policy 14(3):193-203

Pelletier MP, Trépanier M, Morency C (2011) Smart card data use in public transit: A literature review. Transportation Research Part C: Emerging Technologies 19(4):557-568

Rousseeuw PJ (1987) Silhouettes: a graphical aid to the interpretation and validation of cluster analysis. Journal of computational and applied mathematics 20:53-65

Transport for London (2012) Join in the celebration across the capital this summer with a limited edition summer oyster card. Press Release

Utsunomiya M, Attanucci J, Wilson N (2006) Potential uses of transit smart card registration and transaction data to improve transit planning. Transportation Research Record: Journal of the Transportation Research Board 1971(1):119-126

Viggiano C, Koutsopoulos HN, Attanucci J, Wilson NH (2016) Inferring public transport access distance from smart card registration and transaction data. Transportation Research Record: Journal of the Transportation Research Board 2544, forthcoming

Vuchic V, Musso A (1991) Theory and practice of metro network design. Public transport international 40(3/91) 